\title{
A Review of Researches on the Intervention of Problematic Social Media Use (PSMU) by Adolescent
}

\author{
Jian Mao ${ }^{1}$, Jiashun Xie ${ }^{1}$, Jian Wang, ${ }^{2, *}$

\begin{abstract}
${ }^{I}$ School of Humanities, Jiangxi University of Traditional Chinese Medicine, Nan Chang, Jiang Xi, China
*Corresponding author. Email: jwangboy@126.com
\end{abstract} \\ ${ }^{2}$ Mental Health Education Research Center, Nanchang Normal University, Nan Chang, Jiang Xi, China
}

\begin{abstract}
The use of mobile social media has become a part of the daily life of adolescent. While it brings speed, efficiency and convenience to young people's study, work and life, the negative impact of excessive use of mobile social media has also become increasingly prominent. Effective prevention and improvement of problematic social media use (PSMU) behaviors of adolescents have become aspirations of adolescents themselves, their families, schools and society. Paper sorts out the intervention research on problematic social media use behaviors of adolescents, and reviews it from three aspects: self-intervention, individual treatment intervention, and environmental intervention.
\end{abstract}

Keywords: Adolescent, Problematic social media use, Prevention, Intervention.

\section{INTRODUCTION}

PSMU refers to an individual's long-term and highintensity use of mobile social media, which negatively affects physiology, psychology and behavior, and affects normal learning and life to a certain extent, but has not yet reached a pathological level of mobile media use behavior[1]. This behavior has a significant negative impact on adolescents' psychology and behavior, for example, it increases the risk of physical diseases and online injuries, leads to decreased sleep quality and impaired cognitive function, affects their academic performance, and online negative social comparison leads to negative self-evaluation and negative emotional experience. Faced with increasingly prominent problematic social network use behaviors and their negative consequences, teenagers themselves hope to reduce their reliance on social media. Parents are eager for their children to spend time studying and interacting with offline family and friends, while schools are eager children should not affect their normal study life by indulging in social networks. These desires from many sources all hope to alleviate and reduce the negative impact of social networks on young people to a certain extent[2]. Previous studies have shown that personality traits, emotional factors (depression, anxiety), cognitive factors, and psychological motivations are all related to the formation of PSMU by adolescents, and there are complex mechanisms between these factors[3]. In order to explore the effectiveness of interventions to improve the negative effects of problematic social media, and to reflect the value of preliminary basic research.

In recent years, some researchers have actively carried out intervention treatment methods and theoretical research based on previous research results on the formation mechanism of PSMU, filling the gaps in the research on the direction of PSMU behavior intervention. Through reading related literature, it is found that current intervention studies based on PSMU behavior can be divided into three types: Self intervention, Individual therapeutic intervention, Environmental intervention.

\section{SELF-INTERVENTION}

Self-intervention has been proved to be an effective way to treat PSMU. By enhancing self-control ability and reducing the time spent using social media, the dependence on social media can be effectively reduced, and the happiness and learning efficiency of users can be improved[4]. In addition, due to the reduction of time spent using social media, the frequency of outdoor activities of users also increases. In addition, many time management tools (ColdTurkey, 24PI, Timestamp, etc.) have been developed to help social media users better manage their time and use behavior. For example, MSC has developed a mobile time management APP based on reality therapy. This mobile application allows users to 
manage the use of online social networking applications. When users exceed this range, the mobile application suggests replacing the online social network with other activities[5]. And the mobile application also includes the Online Social Network Addiction Scale (Esmaeili Rad \& Ahmadi, 2017) to measure the level of online social network addiction. Comparing the results before and after the intervention group (using the developed mobile APP) with the control group (not using the APP), the researchers found that the development of the mobile APP reduced levels of social network addiction, depression and anxiety, as well as increased individual life satisfaction.

\section{INDIVIDUAL THERAPEUTIC INTERVENTION}

The individual intervention directly acts on the target, aiming to restrain the individual's social media dependence by blocking the transmission channels of social media dependence or replacing the use of social media with certain activities. Individual therapy intervention mainly includes cognitive therapy and exercise therapy.

\subsection{Cognitive Intervention}

Cognitive therapy is mainly through the intervention of inappropriate emotional regulation, to help patients get rid of the wrong cognition, to achieve the purpose of adjusting emotions and reducing addictive behavior. The cognitive maladjustment is an important predictor of adolescents' PSMU behavior, and the lack of selfregulation and emotional regulation by relying on social media are also important reasons for the tendency of social media addiction[6-7]. Therefore, researchers used cognitive therapy to try to amuse the negative consequences of adolescents' PSMU. These methods include cognitive reconstruction, cognitive behavioral therapy, mindfulness cognitive therapy, group counseling and a combination of these methods[8]. For example, Jiang Yongzhi and other studies using mindfulness cognitive therapy to conduct group counseling on PSMU users of college students have shown that individuals who have been trained in mindfulness cognitive training can reduce the time and frequency of mobile social network use through attention control, and improve mobile social network use. The delayed gratification capability used by social networks can improve PSMU behavior[2]. Zhou conducted an intervention study on problematic social media users based on cognitive behavioral therapy and combined with short-term comprehensive intervention methods that reduce the time spent on social media. The research results show that this short-term comprehensive intervention method can improve users' life satisfaction and increase their work efficiency. After the intervention, users have closer offline relationships and stronger autonomy[9]. Wang Xiaoyu conducted a growth group work intervention intervention on 12 college students who are prone to social media addiction[4]. The comparison of the results between the experimental group and the control group showed that after the group activities, the students in the experimental group improved their irrational cognition of self and social media use. The level of social media addiction has decreased. At the same time, it has improved its interpersonal communication and communication skills in real life.

In short, cognitive therapy and comprehensive application have obvious effects on the intervention of PSMU behaviors. The therapist assists the individual to deeply perceive the problem emotions and psychological cognitive process, to further guide the individual to discover their own irrational cognition and behavior, and to assist the individual to have a stronger drive to improve their problem behaviors and develop healthy behaviors. The habit of using social media can improve the happiness and life satisfaction of users.

\subsection{Exercise Therapy}

Previous studies have generally confirmed the intervention effect of exercise therapy on addictive behaviors. The mechanism is that highly excited nerve cells are rested and adjusted after exercise, which enhances the individual's emotional adaptation ability to external changes. The results of scholars in the intervention study of mobile phone dependence show that sports interventions such as volleyball sports and outdoor development training can improve mobile phone dependence behavior[10]. Nowadays, with the widespread popularity of mobile phones, the main way for teenagers to use social media is through mobile phones, and research has also shown that there is an important relationship between mobile phone dependence and PSMU. Therefore, physical exercise intervention may also be effective in improving PSMU behavior, but there is no exercise intervention study on PSMU behavior of adolescents. In addition, exercise therapy requires strong self-control and external supervision, and is easily interfered by other irrelevant factors; once the external supervision is weakened, the effect of exercise therapy may rebound.

\section{ENVIRONMENTAL INTERVENTION}

Environmental intervention is a kind of implicit intervention method, which mainly takes the family, school, and society as the main body to carry out individual intervention or coordinated intervention, 
through parents' words and deeds, school guidance and education, and standardized network management systems[10].The construction method creates a good social atmosphere and enhances the "immunity" of dependence on social media. Home and school are important growth environments for adolescents. As a secondary prevention strategy, family therapy can play an important role in reducing major risk factors, providing emotional support, and reducing excessive use of social media. Parents should strengthen emotional communication with their children, learn more about their children's inner world, and patiently listen to their inner needs. When children are facing difficulties or negative emotions, assist them to feel more warmth and care from the family so as to reduce their dependence on virtual social relationships; By focusing on the norms and policies that promote healthy rather than unhealthy social media use behavior, schools seem to have a certain effect on preventing and improving PSMU. For example, mental health education courses. Studies have shown that mental health education courses based on the influencing factors of PSMU behavior have a positive effect on alleviating individual PSMU behaviors[11]. Therefore, a comprehensive intervention in schools and families can be used as the main treatment and intervention strategy as well as a strategy to prevent recurrence.

\section{CONCLUSION}

Compared with other groups, teenagers prefer to use social networks for communication, which makes it easier for teenagers to form PSMU behaviors[12], and the negative impact on their physical development and psychological behavior is increasingly prominent, making parents, schools and society more affected. troubled. Based on current research, self-intervention, cognitive therapy and its comprehensive application, and environmental intervention can improve the PSMU among adolescents to a certain extent. Teenagers can reduce their social media dependence by enhancing their self-control or using some mobile applications based on time management (24PI, etc.). Cognitive therapy and its comprehensive application are currently the most discussed prevention and intervention methods by researchers.

For example, cognitive behavioral therapy and team mindfulness cognitive training have a positive effect on alleviating PSMU behaviors of adolescents; In addition, the formation of problematic social media use is not only affected by individual psychological factors, but also by environmental factors such as family and society. Family intervention and school intervention are also important ways to prevent and improve problematic social media use by young people. Although studies have shown that these interventions are effective in improving PSMU among young people, the current research still has some shortcomings.

1) At present, there are very few intervention studies on PSMU, and the intervention target is also a small sample. The effectiveness and universality of its intervention methods need to be tested.

2) The treatment sample lacks homogeneity. Different experiments use different problematic diagnosis and evaluation tools, making it difficult to compare and repeat research results;

3) Lack of longitudinal tracking research. These studies are all cross-sectional designs, and do not see long-term longitudinal follow-up studies, so it is difficult to evaluate the long-term treatment effect.

Therefore, in the future, researchers still need to test the effectiveness of these intervention methods. Longitudinal studies can be carried out by taking a larger representative sample and using a unified evaluation tool. In addition, prevention is more important than correction for problematic social media use behaviors of adolescents. Research on the prevention of problematic social media use by adolescents should be vigorously strengthened, At the same time, because young people's problematic social media use is not only an individual's unilateral psychological and behavioral problems, but also the result of the joint influence of many factors such as family, school, and society. If only one aspect of the problem is solved in the treatment, after the PSMU users return to the real environment, other factors are likely to induce this factor again, leading to the recurrence of the problematic use behavior. Therefore, multi-system intervention is carried out from the individual, family, school, and social levels.

\section{REFERENCES}

[1] Y.Z.Jiang, X.L.Bai, Alatan Bagen, et al.Adolescent Problematic Social Network Use [J]. Advances in Psychological Science, 2016,24(9):1435-1447.

[2] Y.Z.Jiang, X.L.Bai, 73. Study of Mindfulness Based Cognitive Therapy on the Problematic Mobile Social Network Usage of College Students [J]. The Chinese Health Service Management, 2020,37(03):219-223.

[3] Y.Z.Jiang. A review on the psychological formation mechanism of adolescents' problematic mobile social media use [J]. Education Research Monthly, 2018(12):45-56.

[4] X.Y Wang. Research on Group Work Intervention in college students with social network addictionTake college students of L university in L city as an example [D]. Lanzhou University, Gansu.2020. 
[5] Esmaeili Rad M, Ahmadi F. A new method to measure and decrease the online social networking addiction. Asia-Pacific Psychiatry. 2018;e12330. https://doi.org/10.1111/appy.12330

[6] Lee Z W Y, Cheung C M K, Thadani D R. An Investigation into the Problematic Use of Facebook, 2012[C]. IEEE, 2012.

[7] Pontes H M, Taylor M, Stavropoulos V. Beyond "Facebook Addiction": The Role of CognitiveRelated Factors and Psychiatric Distress in Social Networking Site Addiction[J]. Cyberpsychol Behav Soc Netw, 2018,21(4):240-247.

[8] Hou, Y., Xiong, D., Jiang, T., Song, L., \& Wang, Q. Social media addiction: Its impact, mediation, and intervention[J]. Cyberpsychology, 2019,13(1). article 4. http://dx.doi.org/10.5817/CP2019-1-4

[9] Zhou X, Rau P P, Yang C, et al. Cognitive Behavioral Therapy-Based Short-Term Abstinence Intervention for Problematic Social Media Use: Improved WellBeing and Underlying Mechanisms[J]. Psychiatric Quarterly, 2020. https://doi.org/10.1007/s11126020-09852-0

[10] M. Zhang, Q. Xiao, L.Y Zhu. Research Progress in Mobile Phone Dependence: Causes, Outcomes and Interventions $[\mathrm{J}]$. Chinese Journal of Special Education, 2019(11):88-96.

[11] X.L.Bai, Y.Z .Jiang, T.L.Jin, et al. Intervention and effect evaluation of problematic mobile social network use by college students[J]. Chinese School Health, 2019,40(02):253-255.

[12] Valkenburg, P. M., \& Peter. Online communication among adolescents: An integrated model of its attraction, opportunities, and risks[J]. Journal of Adolescent Health,2011, 48(2):121-127. 